Research Article

\title{
Solutions and the Generalized Hyers-Ulam-Rassias Stability of a Generalized Quadratic-Additive Functional Equation
}

\author{
M. Janfada and R. Shourvazi \\ Department of Mathematics, Sabzevar Tarbiat Moallem University, P.O. Box 397, Sabzevar, Iran \\ Correspondence should be addressed to M. Janfada, mjanfada@gmail.com
}

Received 23 January 2011; Revised 5 April 2011; Accepted 26 April 2011

Academic Editor: D. Anderson

Copyright (C) 2011 M. Janfada and R. Shourvazi. This is an open access article distributed under the Creative Commons Attribution License, which permits unrestricted use, distribution, and reproduction in any medium, provided the original work is properly cited.

We study general solutions and generalized Hyers-Ulam-Rassias stability of the following $n$ dimensional functional equation $f\left(\sum_{i=1}^{k} x_{i}\right)+(k-2) \sum_{i=1}^{k} f\left(x_{i}\right)=\sum_{i=1}^{k} \sum_{j=1, j>i}^{k} f\left(x_{i}+x_{j}\right), k \geq 3$, on non-Archimedean normed spaces.

\section{Introduction}

In 1960, Ulam [1] proposed the following question: under what conditions does there exist an additive mapping near an approximately additive mapping? Hyers [2] showed that if $X$ and $Y$ are Banach spaces, $\epsilon>0$ and $f: X \rightarrow Y$ are a mapping such that

$$
\|f(x+y)-f(x)-f(y)\|<\epsilon
$$

for all $x, y \in X$, then there exists a unique additive mapping $T: X \rightarrow Y$ such that $\| f(x)-$ $T(x) \|<\epsilon$, for all $x \in X$.

Let $f: X \rightarrow Y$ be a mapping such that $f(t x)$ is continuous in $t \in \mathbb{R}$, for each fixed $x \in X$. Suppose for some $\epsilon>0$ and $p \in[0,1)$,

$$
\|f(x+y)-f(x)-f(y)\|<\epsilon\left(\|x\|^{p}+\|y\|^{p}\right)
$$


for all $x, y \in X$. Rassias [3] showed that there exists a unique $\mathbb{R}$-linear mapping $T: X \rightarrow Y$ such that

$$
\|f(x)-T(x)\|<\frac{2 \epsilon}{2-2^{p}}\|x\|^{p}
$$

for all $x \in X$. Găvruţa [4] generalized the results of Rassias.

The functional equation $f(x+y)+f(x-y)=2 f(x)+2 f(y)$ is called the quadratic functional equation. In particular every solution of the quadratic functional equation is said to be a quadratic mapping (see $[5,6]$ ). It is well known that a mapping $f$ between real vector spaces is quadratic if and only if there exists a unique symmetric biadditive mapping $B$ such that $f(x)=B(x, x)$, for all $x$ (see [7-9]).

A generalized Hyers-Ulam stability problem for the quadratic functional equation was proved by Skof [10] for mappings $f: X \rightarrow Y$, where $X$ is a normed space and $Y$ is a Banach space. Cholewa [11] noticed that the theorem of Skof is still true if the relevant domain $X$ is replaced by an Abelian group. In [12], Czerwik proved the generalized Hyers-Ulam stability of the quadratic functional equation. Borelli and Forti [13] generalized the stability result as follows (cf. $[14,15])$ : let $G$ be an Abelian group and $X$ a Banach space. Assume that a mapping $f: G \rightarrow X$ satisfies the functional inequality

$$
\|f(x+y)+f(x-y)-2 f(x)-2 f(y)\| \leq \varphi(x, y),
$$

for all $x, y \in G$, and $\varphi: G \times G \rightarrow[0, \infty)$ is a function such that

$$
\phi(x, y):=\sum_{i=0}^{\infty} \frac{\varphi\left(2^{i} x, 2^{i} y\right)}{4^{i+1}}<\infty,
$$

for all $x, y \in G$. Then there exists a unique quadratic mapping $Q: G \rightarrow X$ with the property $\|f(x)-Q(x)\| \leq \phi(x, x)$, for all $x \in G$.

Stability of the quadratic functional was also studied by many other authors in various cases (see, e.g., [16-25]).

Let $X$ and $Y$ be some given vector spaces, and let $f: X \rightarrow Y$ be a given mapping. For any $k \geq 3$, define

$$
D f\left(x_{1}, \ldots, x_{k}\right):=f\left(\sum_{i=1}^{k} x_{i}\right)+(k-2) \sum_{i=1}^{k} f\left(x_{i}\right)-\sum_{i=1}^{k} \sum_{j=1, j>i}^{k} f\left(x_{i}+x_{j}\right),
$$

where $x_{i} \in X, i=1, \ldots, k$. One can see that the quadratic function $f: \mathbb{R} \rightarrow \mathbb{R}$ defined by $f(x)=x^{2}$ and any additive mapping satisfy not only the following functional equation:

$$
f(x+y+z)+f(x)+f(y)+f(z)=f(x+y)+f(y+z)+f(z+x)
$$

but also

$$
D f\left(x_{1}, \ldots, x_{k}\right)=0,
$$

for all $x_{i} \in \mathbb{R}$. So it is natural that these functional equations are called quadratic additive. 
The functional equation (1.7) was first solved by Kannappan. In fact he proved that a mapping $f$ on a real vector space is a solution of (1.7) if and only if there exists a symmetric biadditive mapping $B$ and an additive mapping $A$ such that $f(x)=B(x, x)+A(x)$, for any $x$ (see [9]). The stability problem for (1.7) is also studied in [26]. Moreover (1.7) was pexiderized and solved by Kannappan [9].

In [27], solutions and the generalized Hyers-Ulam-Rassias stability of the functional equation (1.8) have been studied for $k=3$.

The generalized Hyers-Ulam-Rassias stability problem for the functional equation (1.8) was first considered by Bae and Park [28]. Also solutions and the generalized UlamGăvruța-Rassias stability of this functional equation were studied by Nakmahachalasint [29]. Indeed for its solutions the following theorem is proved.

Theorem 1.1 (see [29, Theorem 2.1]). Let $n>2$ be a positive integer, and let $X$ and $Y$ be vector spaces. A mapping $f: X \rightarrow Y$ satisfies the functional equation (1.8) if and only if the even part of $f$, defined by $f_{e}(x)=(1 / 2)(f(x)+f(-x))$ for all $x \in X$, satisfies the classical quadratic functional equation and the odd part of $f$, defined by $f_{o}(x)=(1 / 2)(f(x)-f(-x))$ for all $x \in X$, satisfies the Cauchy functional equation $f(x+y)=f(x)+f(y)$.

In Section 2 of this paper, we will prove that the functional equation (1.8) is equivalent to the functional equation (1.7). In Section 3, first we prove the generalized Hyers-UlamRassias stability of the functional equation (1.8) in non-Archimedean normed spaces, and then as a consequence of this result, we prove the generalized Hyers-Ulam-Rassias stability of (1.8) in non-Archimedean normed spaces. Finally using the methods of Theorem 3.1. in [29], directly the generalized Hyers-Ulam-Rassias stability of (1.8) will be proved in non-Archimedean normed spaces. The stability problem in non-Archimedean case has been studied by many authors, for example, see [30-34].

First we need some preliminaries in non-Archimedean normed space.

Let $\mathbb{K}$ be a field. A non-Archimedean absolute value on $\mathbb{K}$ is a function $|\cdot|: \mathbb{K} \rightarrow \mathbb{R}$ such that for any $a, b \in \mathbb{K}$ we have

(i) $|a| \geq 0$ and equality holds if and only if $a=0$,

(ii) $|a b|=|a||b|$,

(iii) $|a+b| \leq \max \{|a|,|b|\}$.

Condition (iii) is called the strong triangle inequality. By (ii), we have $|1|=|-1|=1$. Thus, by induction, it follows from (iii) that $|n| \leq 1$, for each integer $n$. We always assume in addition that $|\cdot|$ is nontrivial, that is,

(iv ) there is an $a_{0} \in \mathbb{K}$ such that $\left|a_{0}\right| \neq 0,1$.

Let $X$ be a linear space over a scalar field $\mathbb{K}$ with a non-Archimedean nontrivial valuation $|\cdot|$. A function $\|\cdot\|: X \rightarrow \mathbb{R}$ is a non-Archimedean norm (valuation) if it is a norm over $\mathbb{K}$ with the strong triangle inequality (ultrametric); namely,

$$
\|x+y\| \leq \max \{\|x\|,\|y\|\}, \quad(x, y \in X)
$$

Then $(X,\|\cdot\|)$ is called a non-Archimedean normed space. 
By a complete non-Archimedean normed space we mean one in which every Cauchy sequence is convergent.

Thanks to the inequality

$$
\left\|x_{n}-x_{m}\right\| \leq \max \left\{\left\|x_{j+1}-x_{j}\right\|: m \leq j \leq n-1\right\}(n>m)
$$

a sequence $\left\{x_{n}\right\}$ is Cauchy if and only if $\left\{x_{n+1}-x_{n}\right\}$ converges to zero in a non-Archimedean space.

\section{Solution of the Functional Equation (1.8)}

Throughout this section, $X$ and $Y$ will be some vector spaces. The following theorem proves that the functional equation (1.8) is equivalent to the functional equation (1.7), and so every solution of the functional equation (1.8) is a summation of a quadratic and an additive mappings.

Theorem 2.1. Let $X$ and $Y$ be common domain and range of the $f^{\prime}$ 's in the functional equations (1.7) and (1.8). Then the functional equation (1.8) is equivalent to (1.7).

Proof. We can easily see that (1.8) implies (1.7). Now, suppose a mapping $f: X \rightarrow Y$ satisfies (1.7), for all $x, y \in X$. Using mathematical induction, we are going to show that, for any $k \geq 3$,

$$
f\left(\sum_{i=1}^{k} x_{i}\right)+(k-2) \sum_{i=1}^{k} f\left(x_{i}\right)=\sum_{i=1}^{k} \sum_{j=1, j>i}^{k} f\left(x_{i}+x_{j}\right) .
$$

Let $M=f\left(\sum_{i=1}^{k-1} x_{i}\right)+(k-3) \sum_{i=1}^{k-1} f\left(x_{i}\right)$, and suppose (2.1) holds for $k-1$, we prove that (2.1) is valid for $k$. Let $x_{1}, x_{2}, \ldots, x_{k} \in X$ be given and $k$ odd. By the assumption of induction, we have

$$
\begin{aligned}
\sum_{i=1}^{k} \sum_{j=1, j>i}^{k} f\left(x_{i}+x_{j}\right) & \\
= & f\left(\sum_{i=1}^{k-1} x_{i}\right)+(k-3) \sum_{i=1}^{k} f\left(x_{i}\right)+\sum_{i=1}^{k-1} f\left(x_{i}+x_{k}\right) \\
= & M+\sum_{i=1}^{(k-1) / 2}\left[f\left(x_{2 i-1}+x_{k}\right)+f\left(x_{2 i}+x_{k}\right)+f\left(x_{2 i-1}+x_{2 i}\right)-f\left(x_{2 i-1}+x_{2 i}\right)\right] \\
= & M+\sum_{i=1}^{(k-1) / 2}\left[f\left(x_{2 i-1}+x_{2 i}+x_{k}\right)+f\left(x_{2 i-1}\right)+f\left(x_{2 i}\right)+f\left(x_{k}\right)\right]-\sum_{i=1}^{(k-1) / 2} f\left(x_{2 i-1}+x_{2 i}\right) \\
= & M+f\left(x_{1}+x_{2}+x_{k}\right)+f\left(x_{3}+x_{4}+x_{k}\right)+f\left(x_{1}+x_{2}+x_{3}+x_{4}\right) \\
& -f\left(x_{1}+x_{2}+x_{3}+x_{4}\right)+\sum_{i=3}^{(k-1) / 2} f\left(x_{2 i-1}+x_{2 i}+x_{k}\right)+\sum_{i=1}^{k-1} f\left(x_{i}\right)+\frac{k-1}{2} f\left(x_{k}\right) \\
& \quad-\sum_{i=1}^{(k-1) / 2} f\left(x_{2 i-1}+x_{2 i}\right)
\end{aligned}
$$




$$
\begin{aligned}
& =M+f\left(x_{k}\right)+f\left(\sum_{i=1}^{4} x_{i}+x_{k}\right)+f\left(x_{1}+x_{2}\right)+f\left(x_{3}+x_{4}\right)+f\left(x_{5}+x_{6}+x_{k}\right) \\
& +f\left(\sum_{i=1}^{6} x_{i}\right)-f\left(\sum_{i=1}^{6} x_{i}\right)-f\left(\sum_{i=1}^{4} x_{i}\right)+\sum_{i=4}^{(k-1) / 2} f\left(x_{2 i-1}+x_{2 i}+x_{k}\right) \\
& +\sum_{i=1}^{k-1} f\left(x_{i}\right)+\frac{k-1}{2} f\left(x_{k}\right)-\sum_{i=1}^{(k-1) / 2} f\left(x_{2 i-1}+x_{2 i}\right) \\
& =M+f\left(x_{k}\right)+f\left(\sum_{i=1}^{4} x_{i}+x_{k}\right)+f\left(x_{5}+x_{6}+x_{k}\right)+f\left(\sum_{i=1}^{6} x_{i}\right)-f\left(\sum_{i=1}^{6} x_{i}\right) \\
& -f\left(\sum_{i=1}^{4} x_{i}\right)+\sum_{i=4}^{(k-1) / 2} f\left(x_{2 i-1}+x_{2 i}+x_{k}\right)+\sum_{i=1}^{k-1} f\left(x_{i}\right)+\frac{k-1}{2} f\left(x_{k}\right)-\sum_{i=3}^{(k-1) / 2} f\left(x_{2 i-1}+x_{2 i}\right) \\
& =M+f\left(x_{k}\right)+f\left(\sum_{i=1}^{6} x_{i}+x_{k}\right)+f\left(\sum_{i=1}^{4} x_{i}\right)+f\left(x_{5}+x_{6}\right)+f\left(x_{k}\right)-f\left(\sum_{i=1}^{6} x_{i}\right) \\
& -f\left(\sum_{i=1}^{4} x_{i}\right)+\sum_{i=4}^{(k-1) / 2} f\left(x_{2 i-1}+x_{2 i}+x_{k}\right)+\sum_{i=1}^{k-1} f\left(x_{i}\right)+\frac{k-1}{2} f\left(x_{k}\right)-\sum_{i=3}^{(k-1) / 2} f\left(x_{2 i-1}+x_{2 i}\right) \\
& =M+2 f\left(x_{k}\right)+f\left(\sum_{i=1}^{6} x_{i}+x_{k}\right)+f\left(x_{7}+x_{8}+x_{k}\right)+f\left(\sum_{i=1}^{8} x_{i}\right)-f\left(\sum_{i=1}^{8} x_{i}\right) \\
& -f\left(\sum_{i=1}^{6} x_{i}\right)+\sum_{i=5}^{(k-1) / 2} f\left(x_{2 i-1}+x_{2 i}+x_{k}\right)+\sum_{i=1}^{k-1} f\left(x_{i}\right)+\frac{k-1}{2} f\left(x_{k}\right)-\sum_{i=4}^{(k-1) / 2} f\left(x_{2 i-1}+x_{2 i}\right) \\
& =M+3 f\left(x_{k}\right)+f\left(\sum_{i=1}^{8} x_{i}+x_{k}\right)+f\left(\sum_{i=1}^{6} x_{i}\right)+f\left(x_{7}+x_{8}\right)-f\left(\sum_{i=1}^{8} x_{i}\right)-f\left(\sum_{i=1}^{6} x_{i}\right) \\
& +\sum_{i=5}^{(k-1) / 2} f\left(x_{2 i-1}+x_{2 i}+x_{k}\right)+\sum_{i=1}^{k-1} f\left(x_{i}\right)+\frac{k-1}{2} f\left(x_{k}\right)-\sum_{i=4}^{(k-1) / 2} f\left(x_{2 i-1}+x_{2 i}\right) \\
& =M+3 f\left(x_{k}\right)+f\left(\sum_{i=1}^{8} x_{i}+x_{k}\right)-f\left(\sum_{i=1}^{8} x_{i}\right)+\sum_{i=5}^{(k-1) / 2} f\left(x_{2 i-1}+x_{2 i}+x_{k}\right) \\
& +\sum_{i=1}^{k-1} f\left(x_{i}\right)+\frac{k-1}{2} f\left(x_{k}\right)-\sum_{i=5}^{(k-1) / 2} f\left(x_{2 i-1}+x_{2 i}\right) \\
& =\cdots=M+\frac{k-3}{2} f\left(x_{k}\right)+f\left(\sum_{i=1}^{k-1} x_{i}+x_{k}\right)-f\left(\sum_{i=1}^{k-1} x_{i}\right)+\sum_{i=1}^{k-1} f\left(x_{i}\right)+\frac{k-1}{2} f\left(x_{k}\right) \\
& =(k-3) \sum_{i=1}^{k-1} f\left(x_{i}\right)+(k-2) f\left(x_{k}\right)+f\left(\sum_{i=1}^{k} x_{i}\right)+\sum_{i=1}^{k-1} f\left(x_{i}\right) \\
& =f\left(\sum_{i=1}^{k} x_{i}\right)+(k-2) \sum_{i=1}^{k} f\left(x_{i}\right) \text {. }
\end{aligned}
$$

The proof of the case that $k$ is even is very similar and is omitted. Thus (1.7) and (1.8) are equivalent. 


\section{Generalized Hyers-Ulam-Rassias Stability of the Functional Equation (1.8)}

In this section, we assume that $X$ and $Y$ are a linear space and a complete non-Archimedean normed space, respectively, over a scalar field $\mathbb{K}$ with a non-Archimedean nontrivial valuation $|\cdot|$.

Lemma 3.1. Assume that a mapping $f: X \rightarrow Y$ satisfies the following inequality:

$$
\|f(x+y+z)+f(x)+f(y)+f(z)-f(x+y)-f(y+z)-f(z+x)\| \leq \varphi(x, y, z),
$$

where $\varphi: X \times X \times X \rightarrow[0, \infty)$ is an arbitrary mapping and $x, y, z \in X$. Then,

$$
\begin{array}{r}
\left\|f(x)-\frac{2^{n}+1}{2^{2 n+1}} f\left(2^{n} x\right)+\frac{2^{n}-1}{2^{2 n+1}} f\left(-2^{n} x\right)\right\| \\
\leq \max \left\{\max \left\{\frac{\left|2^{i+1}-1\right|}{|2|^{2 i+3}}\left(\varphi\left(-2^{i} x,-2^{i} x, 2^{i} x\right),|2| \varphi(0,0,0)\right): 0 \leq i<n\right\},\right. \\
\left.\quad \max \left\{\frac{\left|2^{i+1}+1\right|}{|2|^{2 i+3}}\left(\varphi\left(2^{i} x, 2^{i} x,-2^{i} x\right),|2| \varphi(0,0,0)\right): 0 \leq i<n\right\}\right\},
\end{array}
$$

for all $x \in X$ and $n \in \mathbb{N}$.

Proof. If we replace $x, y$ and $z$ in (3.1) by 0 , we get $\|f(0)\| \leq \varphi(0,0,0)$. Putting $x=y=-z$ in (3.1), it yields

$$
\|3 f(x)+f(-x)-f(2 x)\| \leq \max \{\varphi(x, x,-x),|2| \varphi(0,0,0)\} .
$$

By substituting $-x$ for $x$ in (3.3), we get

$$
\|3 f(-x)+f(x)-f(-2 x)\| \leq \max \{\varphi(-x,-x, x),|2| \varphi(0,0,0)\} .
$$

We use induction on $n$ to prove our claim. By (3.3) and (3.4), we have

$$
\begin{aligned}
\| f(x) & -\left(\frac{3}{8}\right) f(2 x)+\left(\frac{1}{8}\right) f(-2 x) \| \\
& \leq \max \left\{\left|\frac{3}{8}\right|\|3 f(x)+f(-x)-f(2 x)\|,\left|\frac{1}{8}\right|\|-3 f(-x)-f(x)+f(-2 x)\|\right\} \\
& \leq \max \left\{\left|\frac{3}{8}\right|(\varphi(x, x,-x),|2| \varphi(0,0,0)),\left|\frac{1}{8}\right|(\varphi(-x,-x, x),|2| \varphi(0,0,0))\right\} .
\end{aligned}
$$


This proves the validity of the inequality (3.2) for $n=1$. Now, assume that inequality (3.2) holds true for some $n \in \mathbb{N}$. By using (3.3), (3.4), and the following relation

$$
\begin{aligned}
f(x)- & \frac{2^{n+1}+1}{2^{2 n+3}} f\left(2^{n+1} x\right)+\frac{2^{n+1}-1}{2^{2 n+3}} f\left(-2^{n+1} x\right) \\
= & f(x)-\frac{2^{n}+1}{2^{2 n+1}} f\left(2^{n} x\right)+\frac{2^{n}-1}{2^{2 n+1}} f\left(-2^{n} x\right) \\
& +\frac{2^{n+1}+1}{2^{2 n+3}}\left[3 f\left(2^{n} x\right)+f\left(-2^{n} x\right)-f\left(2^{n+1} x\right)\right] \\
& -\frac{2^{n+1}-1}{2^{2 n+3}}\left[3 f\left(-2^{n} x\right)+f\left(2^{n} x\right)-f\left(-2^{n+1} x\right)\right],
\end{aligned}
$$

we get inequality (3.2) for $n+1$. This completes the proof.

In the following two theorems, the generalized Hyers-Ulam-Rassias stability of (1.7) is proved under the approximately even and approximately odd conditions, respectively.

Theorem 3.2. Assume that a mapping $f: X \rightarrow Y$ satisfies the following inequality:

$$
\begin{gathered}
\|f(x+y+z)+f(x)+f(y)+f(z)-f(x+y)-f(y+z)-f(z+x)\| \leq \varphi(x, y, z), \\
\|f(x)-f(-x)\| \leq \psi(x),
\end{gathered}
$$

where $\varphi: X \times X \times X \rightarrow[0, \infty)$ and $\psi: X \rightarrow[0, \infty)$ are mappings such that

$$
\begin{gathered}
\lim _{n \rightarrow \infty} \frac{\varphi\left(2^{n} x, 2^{n} y, 2^{n} z\right)}{|2|^{2 n}}=0, \\
\tilde{\varphi}(x, y, z)=\lim _{n \rightarrow \infty} \max \left\{\frac{\left|2^{i+1}-1\right|}{|2|^{2 i+3}}\left(\varphi\left(2^{i} x, 2^{i} y, 2^{i} z\right),|2| \varphi(0,0,0)\right): 0 \leq i<n\right\}<\infty, \\
\lim _{k \rightarrow \infty} \lim _{n \rightarrow \infty} \max \left\{\frac{\left|2^{i+1}-1\right|}{|2|^{2 i+3}}\left(\varphi\left(2^{i} x, 2^{i} y, 2^{i} z\right),|2| \varphi(0,0,0)\right): k \leq i<n+k\right\}=0, \\
\lim _{n \rightarrow \infty} \frac{\psi\left(2^{n} x\right)}{|2|^{2 n}}=0,
\end{gathered}
$$

for all $x, y, z \in X$. Then there exists a unique quadratic mapping $Q: X \rightarrow Y$ which satisfies (1.7) and the inequality

$$
\|f(x)-Q(x)\| \leq \max \{\tilde{\varphi}(-x,-x, x), \widetilde{\Phi}(x, x,-x)\}
$$


where

$$
\widetilde{\Phi}(x, y, z)=\lim _{n \rightarrow \infty} \max \left\{\frac{\left|2^{i+1}+1\right|}{|2|^{2 i+3}}\left(\varphi\left(2^{i} x, 2^{i} y, 2^{i} z\right),|2| \varphi(0,0,0)\right): 0 \leq i<n\right\},
$$

for all $x, y, z \in X$. If moreover $f$ is measurable or $f(t x)$ is continuous in $t$ for each fixed $x \in X$ then $Q(t x)=t^{2} Q(x)$, for all $x \in X$ and $t \in \mathbb{R}$.

Proof. It follows from (3.2), the second condition in (3.7) and Lemma 3.1, that

$$
\begin{aligned}
& \left\|f(x)-\frac{f\left(2^{n} x\right)}{2^{2 n}}\right\| \\
& =\left\|f(x)-\frac{2^{n}+1}{2^{2 n+1}} f\left(2^{n} x\right)+\frac{2^{n}-1}{2^{2 n+1}} f\left(-2^{n} x\right)-\left(\frac{2^{n}-1}{2^{2 n+1}} f\left(-2^{n} x\right)-\frac{2^{n}-1}{2^{2 n+1}} f\left(2^{n} x\right)\right)\right\| \\
& \leq \max \left\{\max \left\{\frac{\left|2^{i+1}-1\right|}{\mid 2^{2 i+3}}\left(\varphi\left(-2^{i} x,-2^{i} x, 2^{i} x\right),|2| \varphi(0,0,0)\right): 0 \leq i<n\right\},\right. \\
& \left.\max \left\{\frac{\left|2^{i+1}+1\right|}{\mid 2^{2 i+3}}\left(\varphi\left(2^{i} x, 2^{i} x,-2^{i} x\right),|2| \varphi(0,0,0)\right): 0 \leq i<n\right\}, \frac{\left|2^{n}-1\right|}{|2|^{2 n+1}} \psi\left(2^{n} x\right)\right\} .
\end{aligned}
$$

By (3.12), for $n \geq m$, we have

$$
\begin{gathered}
\left\|\frac{f\left(2^{n} x\right)}{2^{2 n}}-\frac{f\left(2^{m} x\right)}{2^{2 m}}\right\| \\
=\frac{1}{|2|^{2 m}}\left\|\frac{1}{2^{2(n-m)}} f\left(2^{n-m} \cdot 2^{m} x\right)-f\left(2^{m} x\right)\right\| \\
\leq \frac{1}{|2|^{2 m}} \max \left\{\max \left\{\frac{\left|2^{i+1}-1\right|}{|2|^{2 i+3}}\left(\varphi\left(-2^{i+m} x,-2^{i+m} x, 2^{i+m} x\right),|2| \varphi(0,0,0)\right): 0 \leq i<n-m\right\},\right. \\
\max \left\{\frac{\left|2^{i+1}+1\right|}{|2|^{2 i+3}}\left(\varphi\left(2^{i+m} x, 2^{i+m} x,-2^{i+m} x\right),|2| \varphi(0,0,0)\right): 0 \leq i<n-m\right\}, \\
\left.\frac{\left|2^{n-m}-1\right|}{\left|2^{2(n-m)+1}\right|} \psi\left(2^{n-m} \cdot 2^{m} x\right)\right\} .
\end{gathered}
$$

Replacing $n, m$ with $n+1, n$, respectively, in (3.13), we can easily get

$$
\begin{aligned}
\left\|\frac{f\left(2^{n+1} x\right)}{2^{2(n+1)}}-\frac{f\left(2^{n} x\right)}{2^{2 n}}\right\| \leq \max \left\{\frac{1}{|2|^{3}}\left(\frac{\varphi\left(-2^{n} x,-2^{n} x, 2^{n} x\right)}{|2|^{2 n}}, \frac{|2| \varphi(0,0,0)}{|2|^{2 n}}\right),\right. \\
\frac{|3|}{|2|^{3}}\left(\frac{\varphi\left(2^{n} x, 2^{n} x,-2^{n} x\right)}{|2|^{2 n}}, \frac{|2| \varphi(0,0,0)}{|2|^{2 n}}\right), \frac{1}{|2|} \frac{\psi\left(2^{n+1} x\right)}{\left.|2|^{2(n+1)}\right\} .}
\end{aligned}
$$


The sequence $\left\{f\left(2^{n} x\right) / 2^{2 n}\right\}$ is a Cauchy sequence, since the right-hand side of the inequality (3.14) tends to 0 as $n$ tends to $\infty$. Therefore, we may apply a direct method for definition of $Q$. Define

$$
Q(x)=\lim _{n \rightarrow \infty} \frac{f\left(2^{n} x\right)}{2^{2 n}}
$$

for all $x \in X$. From the first condition in (3.7), it follows that

$$
\begin{gathered}
\| \frac{f\left(2^{n}(x+y+z)\right)}{2^{2 n}}+\frac{f\left(2^{n} x\right)}{2^{2 n}}+\frac{f\left(2^{n} y\right)}{2^{2 n}}+\frac{f\left(2^{n} z\right)}{2^{2 n}}-\frac{f\left(2^{n}(x+y)\right)}{2^{2 n}} \\
-\frac{f\left(2^{n}(y+z)\right)}{2^{2 n}}-\frac{f\left(2^{n}(z+x)\right)}{2^{2 n}} \| \leq \frac{\varphi\left(2^{n} x, 2^{n} y, 2^{n} z\right)}{\mid 2^{2 n}},
\end{gathered}
$$

for all $x, y, z \in X$ and for all $n \in \mathbb{N}$. Therefore, by letting $n \rightarrow \infty$ in the last inequality, from (3.8), it is clear that $Q$ is a solution of (1.7). Analogously, by the second condition in (3.7), $Q$ is even. By putting $z=-y$ in (1.7) and taking account of $Q(0)=0$, we see $Q$ as an even solution of (1.7) and so is quadratic. According to $|2|^{n} \leq 1$ for all $n \in \mathbb{N}$, we get

$$
\frac{\left|2^{n}-1\right|}{|2|^{2 n+1}} \psi\left(2^{n} x\right) \leq \max \left\{\frac{\left|2^{n}\right|}{|2|^{2 n+1}} \psi\left(2^{n} x\right), \frac{1}{|2|^{2 n+1}} \psi\left(2^{n} x\right)\right\}=\frac{1}{|2|^{2 n+1}} \psi\left(2^{n} x\right) .
$$

Then from (3.12) and definition of $Q$ one may see that inequality (3.10) holds true.

Now, let $T: X \rightarrow Y$ be another quadratic mapping which satisfies (1.7) and inequality (3.10). Obviously, we have

$$
Q\left(2^{n} x\right)=4^{n} Q(x), \quad T\left(2^{n} x\right)=4^{n} T(x),
$$

for all $x \in X$ and $n \in \mathbb{N}$. Hence, it follows from (3.10) that

$$
\begin{aligned}
\|Q(x)-T(x)\| & =|2|^{-2 n}\left\|Q\left(2^{n} x\right)-T\left(2^{n} x\right)\right\| \\
& \leq|2|^{-2 n} \max \left\{\left\|Q\left(2^{n} x\right)-f\left(2^{n} x\right)\right\|,\left\|T\left(2^{n} x\right)-f\left(2^{n} x\right)\right\|\right\} \\
& \leq \max \left\{\frac{\tilde{\varphi}\left(-2^{n} x,-2^{n} x, 2^{n} x\right)}{|2|^{2 n}}, \frac{\tilde{\Phi}\left(2^{n} x, 2^{n} x,-2^{n} x\right)}{|2|^{2 n}}\right\},
\end{aligned}
$$

for all $x \in X$ and $n \in \mathbb{N}$. Applying (3.8) and letting $n \rightarrow \infty$ in the preceding inequality, we immediately conclude the uniqueness of $Q$. The proof of the last assertion in the theorem goes through in the same way as that of Theorem 1 [12]. 
Theorem 3.3. Assume that a mapping $f: X \rightarrow Y$ satisfies the system of inequalities

$$
\begin{gathered}
\|f(x+y+z)+f(x)+f(y)+f(z)\|-f(x+y)-f(y+z)-f(z+x) \| \leq \varphi(x, y, z), \\
\|f(x)+f(-x)\| \leq \psi(x),
\end{gathered}
$$

for all $x, y, z \in X$, where $\varphi: X \times X \times X \rightarrow[0, \infty)$ and $\psi: X \rightarrow[0, \infty)$ are mappings with conditions of Theorem 3.2. Then with the notations of Theorem 3.2, there exists a unique additive mapping $F: X \rightarrow Y$ satisfying the inequality

$$
\|f(x)-F(x)\| \leq \max \{\tilde{\varphi}(-x,-x, x), \widetilde{\Phi}(x, x,-x)\}
$$

for all $x \in X$.

Proof. From (3.2), the second condition in (3.20), and Lemma 3.1, we get

$$
\begin{aligned}
& \left\|f(x)-\frac{f\left(2^{n} x\right)}{2^{n}}\right\| \\
& =\left\|f(x)-\frac{2^{n}+1}{2^{2 n+1}} f\left(2^{n} x\right)+\frac{2^{n}-1}{2^{2 n+1}} f\left(-2^{n} x\right)-\left(\frac{2^{n}-1}{2^{2 n+1}} f\left(-2^{n} x\right)+\frac{2^{n}-1}{2^{2 n+1}} f\left(2^{n} x\right)\right)\right\| \\
& \leq \max \left\{\max \left\{\frac{\left|2^{i+1}-1\right|}{\mid 2^{2 i+3}}\left(\varphi\left(-2^{i} x,-2^{i} x, 2^{i} x\right),|2| \varphi(0,0,0)\right): 0 \leq i<n\right\},\right. \\
& \left.\quad \max \left\{\frac{\left|2^{i+1}+1\right|}{|2|^{2 i+3}}\left(\varphi\left(2^{i} x, 2^{i} x,-2^{i} x\right),|2| \varphi(0,0,0)\right): 0 \leq i<n\right\}, \frac{\left|2^{n}-1\right|}{|2|^{2 n+1}} \psi\left(2^{n} x\right)\right\} .
\end{aligned}
$$

For $n \geq m$, we have

$$
\begin{aligned}
& \left\|\frac{f\left(2^{n} x\right)}{2^{n}}-\frac{f\left(2^{m} x\right)}{2^{m}}\right\| \\
& =\frac{1}{|2|^{m}}\left\|\frac{1}{2^{(n-m)}} f\left(2^{n-m} \cdot 2^{m} x\right)-f\left(2^{m} x\right)\right\| \\
& \leq \frac{1}{|2|^{m}} \max \left\{\max \left\{\frac{\left|2^{i+1}-1\right|}{|2|^{2 i+3}}\left(\varphi\left(-2^{i+m} x,-2^{i+m} x, 2^{i+m} x\right),|2| \varphi(0,0,0)\right): 0 \leq i<n-m\right\},\right. \\
& \max \left\{\frac{\left|2^{i+1}+1\right|}{|2|^{2 i+3}}\left(\varphi\left(2^{i+m} x, 2^{i+m} x,-2^{i+m} x\right),|2| \varphi(0,0,0)\right): 0 \leq i<n-m\right\}, \\
& \left.\frac{\left|2^{n-m}-1\right|}{\left|2^{2(n-m)+1}\right|} \psi\left(2^{n-m} \cdot 2^{m} x\right)\right\} .
\end{aligned}
$$


Therefore,

$$
\begin{aligned}
\left\|\frac{f\left(2^{n+1} x\right)}{2^{n+1}}-\frac{f\left(2^{n} x\right)}{2^{n}}\right\| \leq \max \left\{\frac{1}{|2|^{3}}\left(\frac{\varphi\left(-2^{n} x,-2^{n} x, 2^{n} x\right)}{|2|^{n}}, \frac{|2| \varphi(0,0,0)}{|2|^{n}}\right),\right. \\
\left.\frac{|3|}{|2|^{3}}\left(\frac{\varphi\left(2^{n} x, 2^{n} x,-2^{n} x\right)}{|2|^{n}}, \frac{|2| \varphi(0,0,0)}{|2|^{n}}\right), \frac{1}{|2|^{2}} \frac{\psi\left(2^{n+1} x\right)}{|2|^{n+1}}\right\} .
\end{aligned}
$$

Conditions of $\varphi$ and $\psi$ and $|4| \leq|2|$ imply that sequence $\left\{f\left(2^{n} x\right) / 2^{n}\right\}$ is a Cauchy sequence. Now, for any $x \in X$, define

$$
F(x)=\lim _{n \rightarrow \infty} \frac{f\left(2^{n} x\right)}{2^{n}} .
$$

Similarly, as in the proof of Theorem 3.2, due to (3.20), we may see that the mapping $F$ satisfies (1.7) and is an odd function. By putting $z=-y$ in (1.7), considering the oddness of $F$, and letting $u=x+y, v=x-y$, we get

$$
2 F\left(\frac{u+v}{2}\right)=F(u)+F(v) .
$$

According to [35], the mapping $F$ is additive, since $F(0)=0$. The validity of inequality (3.21) follows directly from (3.22) and the definition of $F$. Now, let $G: X \rightarrow Y$ be another additive mapping which satisfies (3.21). It then follows from (3.21) that

$$
\begin{aligned}
\|F(x)-G(x)\| & =|2|^{-n}\left\|F\left(2^{n} x\right)-G\left(2^{n} x\right)\right\| \\
& \leq|2|^{-n} \max \left\{\left\|F\left(2^{n} x\right)-f\left(2^{n} x\right)\right\|,\left\|G\left(2^{n} x\right)-f\left(2^{n} x\right)\right\|\right\} \\
& \leq \max \left\{\frac{\tilde{\varphi}\left(-2^{n} x,-2^{n} x, 2^{n} x\right)}{|2|^{n}}, \frac{\widetilde{\Phi}\left(2^{n} x, 2^{n} x,-2^{n} x\right)}{|2|^{n}}\right\},
\end{aligned}
$$

for all $x \in X$ and $n \in \mathbb{N}$. This implies the uniqueness of $F$.

Remark 3.4. The approximately even condition guarantees the quadratic property of $Q$, whereas the approximately odd condition guarantees the additive behavior of $F$.

Corollary 3.5. Let $k \in \mathbb{N}$ and $k \geq 3$. Assume that a mapping $f: X^{k} \rightarrow Y$ satisfies the following inequalities:

$$
\begin{gathered}
\left\|f\left(\sum_{i=1}^{k} x_{i}\right)+(k-2) \sum_{i=1}^{k} f\left(x_{i}\right)-\sum_{i=1}^{k} \sum_{j=1, j>i}^{k} f\left(x_{i}+x_{j}\right)\right\| \leq \phi\left(x_{1}, \ldots, x_{k}\right), \\
\|f(x)-f(-x)\| \leq \psi(x),
\end{gathered}
$$


where $\phi: X^{k} \rightarrow[0, \infty)$ and $\psi: X \rightarrow[0, \infty)$ are mappings such that

$$
\begin{gathered}
\lim _{n \rightarrow \infty} \frac{\phi\left(2^{n} x, 2^{n} y, 2^{n} z, 0, \ldots, 0\right)}{|2|^{2 n}}=0, \\
\tilde{\varphi}(x, y, z)=\lim _{n \rightarrow \infty} \max \left\{\frac{\left|2^{i+1}-1\right|}{|2|^{2 i+3}}\left(\phi\left(2^{i} x, 2^{i} y, 2^{i} z, 0, \ldots, 0\right),|2| \phi(0, \ldots, 0)\right): 0 \leq i<n\right\}<\infty, \\
\lim _{k \rightarrow \infty} \lim _{n \rightarrow \infty} \max \left\{\frac{\left|2^{i+1-k}-1\right|}{|2|^{2 i+3}}\left(\phi\left(2^{i} x, 2^{i} y, 2^{i} z, 0, \ldots, 0\right),|2| \phi(0, \ldots, 0)\right): k \leq i<n+k\right\}=0, \\
\lim _{n \rightarrow \infty} \frac{\psi\left(2^{n} x\right)}{|2|^{2 n}}=0,
\end{gathered}
$$

for all $x, y, z \in X$. Then there exists a unique quadratic mapping $Q: X \rightarrow Y$ which satisfies (1.8) and the following inequality:

$$
\|f(x)-Q(x)\| \leq \max \{\tilde{\varphi}(-x,-x, x), \tilde{\Phi}(x, x,-x)\}
$$

where

$$
\begin{aligned}
& \varphi(x, y, z)=\max \left\{\phi(x, y, z, 0, \ldots, 0), \frac{|2(k-4)(k-3)|}{|(k-2)(k-1)|} \phi(0, \ldots, 0)\right\}, \\
& \tilde{\varphi}(x, y, z)=\lim _{n \rightarrow \infty} \max \left\{\frac{\left|2^{i+1}-1\right|}{|2|^{2 i+3}}\left(\varphi\left(2^{i} x, 2^{i} y, 2^{i} z\right),|2| \varphi(0,0,0)\right), 0 \leq i<n\right\}, \\
& \tilde{\Phi}(x, y, z)=\lim _{n \rightarrow \infty} \max \left\{\frac{\left|2^{i+1}+1\right|}{|2|^{2 i+3}}\left(\phi\left(2^{i} x, 2^{i} y, 2^{i} z,\right),|2| \phi(0,0,0)\right), 0 \leq i<n\right\} .
\end{aligned}
$$

Proof. By letting $x_{i}=0, i=1, \ldots, k$, in (3.28) we get

$$
\|f(0)\| \leq \frac{|2|}{|(k-1)(k-2)|} \phi(0, \ldots, 0),
$$

and also from (3.28) and (3.33) we have

$$
\begin{gathered}
\left\|f\left(x_{1}+x_{2}+x_{3}\right)+f\left(x_{1}\right)+f\left(x_{2}\right)+f\left(x_{3}\right)-f\left(x_{1}+x_{2}\right)-f\left(x_{2}+x_{3}\right)\right\| \\
\leq \max \left\{\phi\left(x_{1}, x_{2}, x_{3}, 0, \ldots, 0\right), \frac{|2(k-4)(k-3)|}{|(k-2)(k-1)|} \phi(0, \ldots, 0)\right\} .
\end{gathered}
$$

Now by considering $\varphi(x, y, z)=\max \{\phi(x, y, z, 0, \ldots, 0),(|2(k-4)(k-3)| /|(k-2)(k-1)|) \phi(0$, $\ldots, 0)\}$, we may see that $\varphi$ satisfies (3.8), and so using Theorem 3.2, we get (3.31). 
Remark 3.6. In the previous corollary, if one replaces (3.29) with

$$
\|f(x)+f(-x)\| \leq \psi(x),
$$

where $\psi: X \rightarrow[0, \infty)$ is a mapping satisfying

$$
\lim _{n \rightarrow \infty} \frac{\psi\left(2^{n} x\right)}{|2|^{2 n}}=0
$$

then using Theorem 3.3 and a similar argument with Corollary 3.5, we may find a unique additive mapping $F: X \rightarrow Y$ which satisfies (1.8) and the inequality

$$
\|f(x)-F(x)\| \leq \max \{\tilde{\varphi}(-x,-x, x), \widetilde{\Phi}(x, x,-x)\}
$$

In the following theorem, using [29] Theorem 3.1, the generalized Hyers-Ulam-Rassias stability of (1.8) is proved directly in non-Archimedean normed spaces. Note that the conditions on $\phi$ in this theorem are different from the previous conditions.

Theorem 3.7. Let $k>2$ be a positive integer, $X$ a non-Archimedean vector space, and $Y$ a non-Archimedean Banach space. Let $\phi: X^{k} \rightarrow[0, \infty)$ be an even function. Define $\varphi(x)=$ $\phi(x, x,-x, 0, \ldots, 0)$ for all $x \in X$. If

$$
\begin{gathered}
\lim _{m \rightarrow \infty} \frac{\phi\left(2^{m} x_{1}, 2^{m} x_{2}, \ldots, 2^{m} x_{k}\right)}{|4|^{m}}=0, \\
\tilde{\varphi}(x)=\lim _{m \rightarrow \infty} \max \left\{\frac{\varphi\left(2^{i} x\right)}{|4|^{i}}: 0 \leq i<m\right\}<\infty, \\
\lim _{k \rightarrow \infty} \lim _{m \rightarrow \infty} \max \left\{\frac{\varphi\left(2^{i} x\right)}{|4|^{i}}: k \leq i<m+k\right\}=0,
\end{gathered}
$$

or

$$
\begin{gathered}
\lim _{m \rightarrow \infty}|2|^{m} \phi\left(\frac{x_{1}}{2^{m}}, \frac{x_{2}}{2^{m}}, \ldots, \frac{x_{k}}{2^{m}}\right)=0, \\
\tilde{\varphi}(x)=\lim _{m \rightarrow \infty} \max \left\{|2|^{i} \varphi\left(\frac{x}{2^{i}}\right): 1 \leq i<m+1\right\}<\infty, \\
\lim _{k \rightarrow \infty} \lim _{m \rightarrow \infty} \max \left\{|2|^{i} \varphi\left(\frac{x}{2^{i}}\right): 1+k \leq i<m+k+1\right\}=0,
\end{gathered}
$$

for all $x_{1}, x_{2}, \ldots, x_{k} \in X$, and a mapping $f: X \rightarrow Y$ satisfies $f(0)=0$ and

$$
\left\|D f\left(x_{1}, \ldots, x_{k}\right)\right\| \leq \phi\left(x_{1}, \ldots, x_{k}\right),
$$


for all $x_{1}, x_{2}, \ldots, x_{k} \in X$, then there exists a unique function $T: X \rightarrow Y$ that satisfies functional equation (1.8), and if condition (3.38) holds,

$$
\begin{gathered}
\left\|f_{e}(x)-T_{e}(x)\right\| \leq \frac{1}{|2|^{3}} \tilde{\varphi}(x), \\
\left\|f_{o}(x)-T_{o}(x)\right\| \leq \frac{1}{|2|^{2}} \tilde{\varphi}_{1}(x),
\end{gathered}
$$

where $\tilde{\varphi}_{1}(x)=\lim _{m \rightarrow \infty} \max \left\{\varphi\left(2^{i} x\right) /|2|^{i}: 0 \leq i<m\right\}$ or, if condition (3.39) holds,

$$
\begin{gathered}
\left\|f_{e}(x)-T_{e}(x)\right\| \leq \frac{1}{|2|^{3}} \tilde{\varphi}(x), \\
\left\|f_{o}(x)-T_{o}(x)\right\| \leq \frac{1}{|2|^{2}} \tilde{\varphi}_{2}(x),
\end{gathered}
$$

where $\tilde{\varphi}_{2}(x)=\lim _{m \rightarrow \infty} \max \left\{|2|^{i} \varphi\left(x / 2^{i}\right): 1 \leq i<m+1\right\}$. The function $T$ is given by

$$
T(x)= \begin{cases}\lim _{m \rightarrow \infty} 4^{-m} f_{e}\left(2^{m} x\right)+2^{-m} f_{o}\left(2^{m} x\right) & \text { if conditions (3.38) hold } \\ \lim _{m \rightarrow \infty} 4^{m} f_{e}\left(2^{-m} x\right)+2^{m} f_{o}\left(2^{-m} x\right) & \text { if conditions (3.39) hold }\end{cases}
$$

for all $x \in X$.

Proof. We will prove the theorem for a function $\phi$ satisfying condition (3.38) and accordingly inequalities (3.41) and (3.42). A proof for conditions (3.39) and inequalities (3.43) and (3.44) can be reproduced in a similar manner. Setting $\left(x_{1}, x_{2}, \ldots, x_{k}\right)=(x, x,-x, 0,0, \ldots, 0)$ in $(3.40)$ and simplifying, we have

$$
\|3 f(x)+f(-x)-f(2 x)\| \leq \varphi(x) .
$$

Replacing $x$ by $-x$, we get

$$
\|3 f(-x)+f(x)-f(-2 x)\| \leq \varphi(-x)=\varphi(x) .
$$

Then,

$$
\begin{aligned}
\left\|4 f_{e}(x)-f_{e}(2 x)\right\| & =\frac{1}{|2|}\|(3 f(x)+f(-x)-f(2 x))+(3 f(-x)+f(x)-f(-2 x))\| \\
& \leq \max \left\{\frac{1}{|2|}\|3 f(x)+f(-x)-f(2 x)\|, \frac{1}{|2|}\|3 f(-x)+f(x)-f(-2 x)\|\right\} \\
& \leq \frac{\varphi(x)}{|2|}
\end{aligned}
$$




$$
\begin{aligned}
\left\|2 f_{o}(x)-f_{o}(2 x)\right\| & =\frac{1}{|2|}\|(3 f(x)+f(-x)-f(2 x))-(3 f(-x)+f(x)-f(-2 x))\| \\
& \leq \max \left\{\frac{1}{|2|}\|3 f(x)+f(-x)-f(2 x)\|, \frac{1}{|2|}\|3 f(-x)+f(x)-f(-2 x)\|\right\} \\
& \leq \frac{\varphi(x)}{|2|} .
\end{aligned}
$$

Rewrite the inequality on $f_{e}$ as $\left\|f_{e}(x)-f_{e}(2 x) / 4\right\| \leq\left(1 /|2|^{3}\right) \varphi(x)$ for all $x \in X$. Then by replacing $x$ by $2^{m} x$ and dividing both sides by $4^{m}$ we have

$$
\left\|\frac{f_{e}\left(2^{m} x\right)}{4^{m}}-\frac{f_{e}\left(2^{m+1} x\right)}{4^{m+1}}\right\| \leq \frac{1}{|2|^{3}} \frac{\varphi\left(2^{m} x\right)}{|4|^{m}} .
$$

Thus

$$
\left\|f_{e}(x)-\frac{f_{e}\left(2^{m} x\right)}{4^{m}}\right\| \leq \frac{1}{|2|^{3}} \max \left\{\frac{\varphi\left(2^{i} x\right)}{|4|^{i}}: 0 \leq i<m\right\},
$$

for every positive integer $m$. If we rewrite the inequality for $f_{o}$ as $\left\|f_{o}(x)-f_{o}(2 x) / 2\right\| \leq$ $\left(1 /|2|^{2}\right) \varphi(x)$ and repeat the same steps as in the case of $f_{e}$, we will have

$$
\begin{gathered}
\left\|\frac{f_{o}\left(2^{m} x\right)}{2^{m}}-\frac{f_{o}\left(2^{m+1} x\right)}{2^{m+1}}\right\| \leq \frac{1}{|2|^{2}} \frac{\varphi\left(2^{m} x\right)}{|2|^{m}}, \\
\left\|f_{o}(x)-\frac{f_{o}\left(2^{m} x\right)}{2^{m}}\right\| \leq \frac{1}{|2|^{2}} \max \left\{\frac{\varphi\left(2^{i} x\right)}{|2|^{i}}: 0 \leq i<m\right\},
\end{gathered}
$$

for every positive integer $m$. By definition of $\phi$, relations (3.49) and (3.38), the sequence $\left\{f_{e}\left(2^{m} x\right) / 4^{m}\right\}$ is a Cauchy sequence in a Banach space. Let $T_{e}(x)=\lim _{m \rightarrow \infty}\left(f_{e}\left(2^{m} x\right) / 4^{m}\right)$ for all $x \in X$, thus

$$
\left\|f_{e}(x)-T_{e}(x)\right\| \leq \frac{1}{|2|^{3}} \lim _{m \rightarrow \infty} \max \left\{\frac{\varphi\left(2^{i} x\right)}{|4|^{i}}: 0 \leq i<m\right\} .
$$

By similar way we have

$$
\left\|f_{o}(x)-T_{o}(x)\right\| \leq \frac{1}{|2|^{2}} \lim _{m \rightarrow \infty} \max \left\{\frac{\varphi\left(2^{i} x\right)}{|2|^{i}}: 0 \leq i<m\right\} .
$$


Define $T(x)=T_{e}(x)+T_{o}(x)$, for all $x \in X$. In order to show that $T$ satisfies (1.8), it is enough to show that $T_{e}$ and $T_{o}$ satisfy (1.8). For convenience, define $D f_{e}$ and $D f_{o}$ as the even part and the odd part of $D f$ in (1.8), respectively. For $T_{e}$, consider

$$
\begin{aligned}
\frac{1}{|4|^{m}}\left\|D f_{e}\left(2^{m} x_{1}, \ldots, 2^{m} x_{k}\right)\right\| & =\frac{1}{|4|^{m}|2|}\left\|D f\left(2^{m} x_{1}, \ldots, 2^{m} x_{k}\right)+D f\left(-2^{m} x_{1}, \ldots,-2^{m} x_{k}\right)\right\| \\
& \leq \frac{1}{|4|^{m}|2|} \phi\left(2^{m} x_{1}, \ldots, 2^{m} x_{k}\right) .
\end{aligned}
$$

As $m$ tend to infinity, the left-hand side approaches $D T_{e}\left(x_{1}, \ldots, x_{k}\right)$ and by conditions (3.38), the right-hand side approaches 0 . Thus, $D T_{e}\left(x_{1}, \ldots, x_{k}\right)=0$. A similar argument shows that $D T_{o}\left(x_{1}, \ldots, x_{k}\right)=0$ since

$$
\lim _{m \rightarrow \infty} \frac{1}{|2|^{m}} \phi\left(2^{m} x_{1}, \ldots, 2^{m} x_{k}\right)=0
$$

Hence, $T=T_{e}+T_{o}$ satisfies (1.8) as desired. Now using Theorem 1.1, one can see that $T_{e}$ and $T_{o}$ are quadratic and additive, respectively. Also letting $m \rightarrow \infty$ in (3.50) one may obtain (3.43).

To prove the uniqueness of $T$, suppose that there exists another function $S: X \rightarrow Y$ that satisfies (1.8) and inequalities (3.41) and (3.42) with $S$ instead of $T$. Then,

$$
\|S(x)-T(x)\| \leq \max \left\{\left\|S_{e}(x)-T_{e}(x)\right\|,\left\|S_{o}(x)-T_{o}(x)\right\|\right\} .
$$

It is straightforward to show that every solution of the quadratic functional equation $f(x+$ $y)+f(x-y)=2 f(x)+2 f(y)$ has the quadratic property $f(n x)=n^{2} f(x)$ and every solution of the linear functional equation $f(x+y)=f(x)+f(y)$ has the linear property $f(n x)=n f(x)$ for every positive integer $\mathrm{n}$ and for every $x$ in the domain. We thus obtain

$$
\begin{aligned}
\|S(x)-T(x)\| & \leq \lim _{m \rightarrow \infty} \max \left\{\frac{1}{|4|^{m}}\left\|S_{e}\left(2^{m} x\right)-f_{e}\left(2^{m} x\right)\right\|, \frac{1}{|2|^{m}}\left\|S_{o}\left(2^{m} x\right)-f_{o}\left(2^{m} x\right)\right\|\right\} \\
& \leq \lim _{m \rightarrow \infty} \max \left\{\frac{1}{|2|^{3}|4|^{m}} \tilde{\varphi}\left(2^{m} x\right), \frac{1}{|2|^{2}|2|^{m}} \widetilde{\varphi}_{1}\left(2^{m} x\right)\right\},
\end{aligned}
$$

for all $x \in X$. This completes the proof.

Corollary 3.8. If $|2|<1$, a mapping $f: X \rightarrow Y$ satisfies $f(0)=0$ and the inequality

$$
\left\|D f\left(x_{1}, x_{2}, \ldots, x_{k}\right)\right\| \leq \epsilon,
$$

for some $\epsilon>0$ and for all $x_{1}, x_{2}, \ldots, x_{k} \in X$, then there exists a unique mapping $T: X \rightarrow Y$ that satisfies functional equation (1.8) and for all $x \in X$,

$$
\left\|f_{e}(x)-T_{e}(x)\right\| \leq \frac{\epsilon}{|2|}, \quad\left\|f_{o}(x)-T_{o}(x)\right\| \leq \frac{\epsilon}{|2|} .
$$


Proof. Let $\phi\left(x_{1}, x_{2}, \ldots, x_{k}\right)=\epsilon$. Then condition (3.39) in Theorem 3.7 holds. Hence, it follows from Theorem 3.7 that there exists a unique mapping $T: X \rightarrow Y$ such that

$$
\begin{aligned}
& \left\|f_{e}(x)-T_{e}(x)\right\| \leq \frac{1}{|2|^{3}} \lim _{m \rightarrow \infty} \max \left\{|4|^{i} \varphi\left(\frac{x}{2^{i}}\right): 1 \leq i<m+1\right\}=\frac{1}{|2|^{3}}|4| \epsilon, \\
& \left\|f_{o}(x)-T_{o}(x)\right\| \leq \frac{1}{|2|^{3}} \lim _{m \rightarrow \infty} \max \left\{|2|^{i} \varphi\left(\frac{x}{2^{i}}\right): 1 \leq i<m+1\right\}=\frac{1}{|2|^{2}}|2| \epsilon .
\end{aligned}
$$

Corollary 3.9. Let $p$ be a positive real number with $0<p<1$ or $p>2$ and $|2|<1$. If a mapping $f: X \rightarrow Y$ satisfies the inequality,

$$
\left\|D f\left(x_{1}, x_{2}, \ldots, x_{k}\right)\right\| \leq \epsilon \sum_{i=1}^{n}\left\|x_{i}\right\|^{p},
$$

for some $\epsilon>0$ and for all $x_{1}, x_{2}, \ldots, x_{k} \in X$, then there exists a unique mapping $T: X \rightarrow Y$ that satisfies functional equation (1.8) and for all $x \in X$,

$$
\begin{gathered}
\left\|f_{e}(x)-T_{e}(x)\right\| \leq \frac{3 \epsilon}{|2|^{3}}\|x\|^{p}, \quad\left\|f_{o}(x)-T_{o}(x)\right\| \leq \frac{3 \epsilon}{|2|^{2}}\|x\|^{p}, \quad \text { for } p>2, \\
\left\|f_{e}(x)-T_{e}(x)\right\| \leq \frac{3 \epsilon}{|2|^{p+1}}\|x\|^{p}, \quad\left\|f_{o}(x)-T_{o}(x)\right\| \leq \frac{3 \epsilon}{|2|^{p+1}}\|x\|^{p}, \quad \text { for } 0<p<1 .
\end{gathered}
$$

Proof. Substituting $x_{1}=x_{2}=\cdots=x_{k}=0$ into (3.62), we get

$$
f(0)+(k-2) k f(0)=\left(\begin{array}{l}
n \\
2
\end{array}\right) f(0) .
$$

It follows that $1+k(k-2)>\left(\begin{array}{c}k \\ 2\end{array}\right)$, since $k>2$. Hence, $f(0)=0$. Let $\phi\left(x_{1}, x_{2}, \ldots, x_{n}\right)=$ $\epsilon \sum_{i=1}^{n}\left\|x_{i}\right\|^{p}$. If $0<p<1$, then condition (3.39) in Theorem 3.7 holds, and it follows that

$$
\begin{aligned}
& \left\|f_{e}(x)-T_{e}(x)\right\| \leq \frac{1}{|2|^{3}}\left(|4| \epsilon\left(\left\|\frac{x}{2}\right\|^{p}+\left\|\frac{x}{2}\right\|^{p}+\left\|\frac{-x}{2}\right\|^{p}\right)\right)=\frac{3 \epsilon}{|2|^{p+1}}\|x\|^{p}, \\
& \left\|f_{o}(x)-T_{o}(x)\right\| \leq \frac{1}{|2|^{2}}\left(|2| \epsilon\left(\left\|\frac{x}{2}\right\|^{p}+\left\|\frac{x}{2}\right\|^{p}+\left\|\frac{-x}{2}\right\|^{p}\right)\right)=\frac{3 \epsilon}{|2|^{p+1}}\|x\|^{p} .
\end{aligned}
$$

If $p>2$, we apply Theorem 3.7 with condition (3.38) to get a similar result.

\section{Acknowledgment}

The authors would like to appreciate the referees for their contributions and their very useful suggestions. 


\section{References}

[1] S. M. Ulam, Problems in Modern Mathematics, John Wiley \& Sons, New York, NY, USA, 1960.

[2] D. H. Hyers, "On the stability of the linear functional equation," Proceedings of the National Academy of Sciences of the United States of America, vol. 27, pp. 222-224, 1941.

[3] Th. M. Rassias, "On the stability of the linear mapping in Banach spaces," Proceedings of the American Mathematical Society, vol. 72, no. 2, pp. 297-300, 1978.

[4] P. Găvruţa, "A generalization of the Hyers-Ulam-Rassias stability of approximately additive mappings," Journal of Mathematical Analysis and Applications, vol. 184, no. 3, pp. 431-436, 1994.

[5] Th. M. Rassias, Functional Equations and Inequalities, vol. 518 of Mathematics and Its Applications, Kluwer Academic Publishers, Dordrecht, The Netherlands, 2000.

[6] Th. M. Rassias, "On the stability of the quadratic functional equation and its applications," Studia Universitatis Babeş-Bolyai, vol. 43, no. 3, pp. 89-124, 1998.

[7] J. Aczél and J. Dhombres, Functional Equations in Several Variables, vol. 31 of Encyclopedia of Mathematics and Its Applications, Cambridge University Press, Cambridge, UK, 1989.

[8] D. H. Hyers, G. Isac, and Th. M. Rassias, Stability of Functional Equations in Several Variables, vol. 34 of Progress in Nonlinear Differential Equations and Their Applications, Birkhäuser, Boston, Mass, USA, 1998.

[9] P. Kannappan, "Quadratic functional equation and inner product spaces," Results in Mathematics, vol. 27, no. 3-4, pp. 368-372, 1995.

[10] F. Skof, "Local properties and approximation of operators," Rendiconti del Seminario Matematico e Fisico di Milano, vol. 53, pp. 113-129, 1983.

[11] P. W. Cholewa, "Remarks on the stability of functional equations," Aequationes Mathematicae, vol. 27, no. 1-2, pp. 76-86, 1984.

[12] St. Czerwik, "On the stability of the quadratic mapping in normed spaces," Abhandlungen aus dem Mathematischen Seminar der Universität Hamburg, vol. 62, pp. 59-64, 1992.

[13] C. Borelli and G. L. Forti, “On a general Hyers-Ulam stability result," International Journal of Mathematics and Mathematical Sciences, vol. 18, no. 2, pp. 229-236, 1995.

[14] C.-G. Park, "Generalized quadratic mappings in several variables," Nonlinear Analysis: Theory, Methods \& Applications, vol. 57, no. 5-6, pp. 713-722, 2004.

[15] C.-G. Park, "On the stability of the quadratic mapping in Banach modules," Journal of Mathematical Analysis and Applications, vol. 276, no. 1, pp. 135-144, 2002.

[16] D. H. Hyers and Th. M. Rassias, "Approximate homomorphisms," Aequationes Mathematicae, vol. 44, no. 2-3, pp. 125-153, 1992.

[17] S.-M. Jung, “On the Hyers-Ulam-Rassias stability of a quadratic functional equation," Journal of Mathematical Analysis and Applications, vol. 232, no. 2, pp. 384-393, 1999.

[18] S.-M. Jung, "Quadratic functional equations of Pexider type," International Journal of Mathematics and Mathematical Sciences, vol. 24, no. 5, pp. 351-359, 2000.

[19] S.-M. Jung, "Stability of the quadratic equation of Pexider type," Abhandlungen aus dem Mathematischen Seminar der Universität Hamburg, vol. 70, pp. 175-190, 2000.

[20] P. Kannappan, Functional Equations and Inequalities with Applications, Springer Monographs in Mathematics, Springer, New York, NY, USA, 2009.

[21] A. K. Mirmostafaee and M. S. Moslehian, "Fuzzy almost quadratic functions," Results in Mathematics, vol. 52, no. 1-2, pp. 161-177, 2008.

[22] M. Mirzavaziri and M. S. Moslehian, "A fixed point approach to stability of a quadratic equation," Bulletin of the Brazilian Mathematical Society, vol. 37, no. 3, pp. 361-376, 2006.

[23] M. S. Moslehian, K. Nikodem, and D. Popa, "Asymptotic aspect of the quadratic functional equation in multi-normed spaces," Journal of Mathematical Analysis and Applications, vol. 355, no. 2, pp. 717-724, 2009.

[24] M. S. Moslehian, "On the orthogonal stability of the Pexiderized quadratic equation," Journal of Difference Equations and Applications, vol. 11, no. 11, pp. 999-1004, 2005.

[25] Th. M. Rassias, "On the stability of functional equations and a problem of Ulam," Acta Applicandae Mathematicae, vol. 62, no. 1, pp. 23-130, 2000.

[26] S.-M. Jung, "On the Hyers-Ulam stability of the functional equations that have the quadratic property," Journal of Mathematical Analysis and Applications, vol. 222, no. 1, pp. 126-137, 1998.

[27] S.-M. Jung, "Quadratic functional equations of Pexider type," International Journal of Mathematics and Mathematical Sciences, vol. 24, no. 5, pp. 351-359, 2000.

[28] J.-H. Bae and W.-G. Park, "On stability of a functional equation with $n$ variables," Nonlinear Analysis: Theory, Methods E Applications, vol. 64, no. 4, pp. 856-868, 2006. 
[29] P. Nakmahachalasint, "On the generalized Ulam-Gavruta-Rassias stability of mixed-type linear and Euler-Lagrange-Rassias functional equations," International Journal of Mathematics and Mathematical Sciences, vol. 2007, Article ID 63239, 10 pages, 2007.

[30] M. Eshaghi Gordji and M. B. Savadkouhi, "Stability of cubic and quartic functional equations in nonArchimedean spaces," Acta Applicandae Mathematicae, vol. 110, no. 3, pp. 1321-1329, 2010.

[31] A. K. Mirmostafaee, "Non-Archimedean stability of quadratic equations," Fixed Point Theory, vol. 11, no. 1, pp. 67-75, 2010.

[32] A. K. Mirmostafaee, "Stability of quartic mappings in non-Archimedean normed spaces," Kyungpook Mathematical Journal, vol. 49, no. 2, pp. 289-297, 2009.

[33] M. S. Moslehian and Th. M. Rassias, "Stability of functional equations in non-Archimedean spaces," Applicable Analysis and Discrete Mathematics, vol. 1, no. 2, pp. 325-334, 2007.

[34] Gh. Sadeghi, R. Saadati, M. Janfada, and J. M. Rassias, "Stability of Euler-Lagrange quadratic functional equations in Non-Archimedean normed spaces," to appear in Hacettepe Journal of Mathematics and Statistics.

[35] J. C. Parnami and H. L. Vasudeva, “On Jensen's functional equation," Aequationes Mathematicae, vol. 43, no. 2-3, pp. 211-218, 1992. 


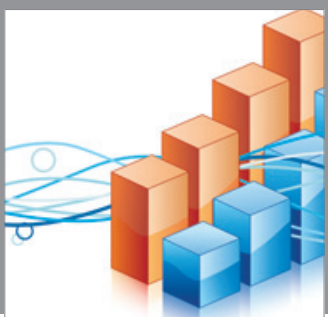

Advances in

Operations Research

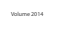

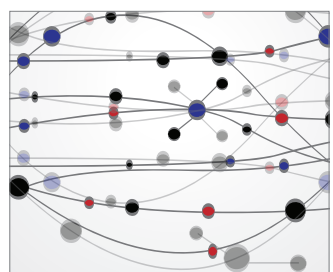

\section{The Scientific} World Journal
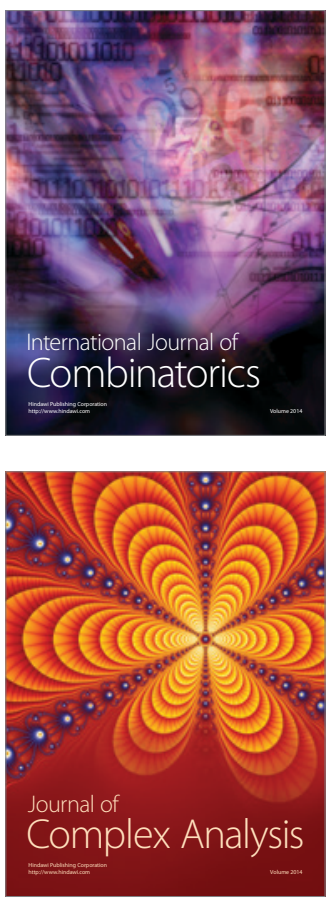

International Journal of

Mathematics and

Mathematical

Sciences
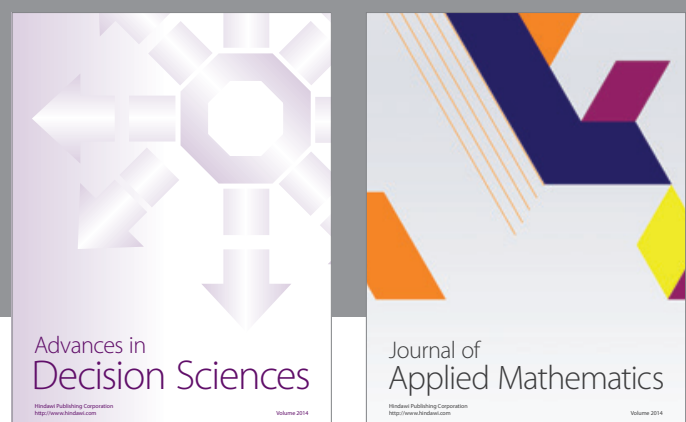

Journal of

Applied Mathematics
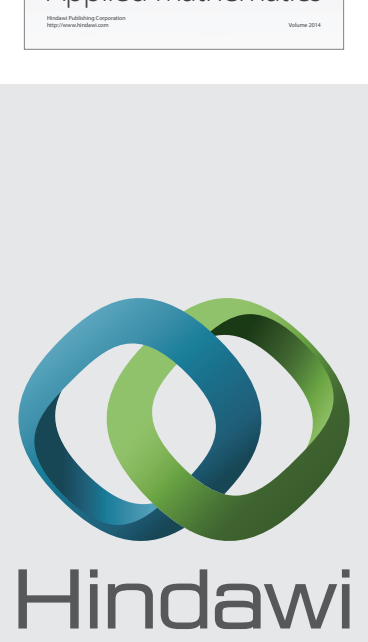

Submit your manuscripts at http://www.hindawi.com
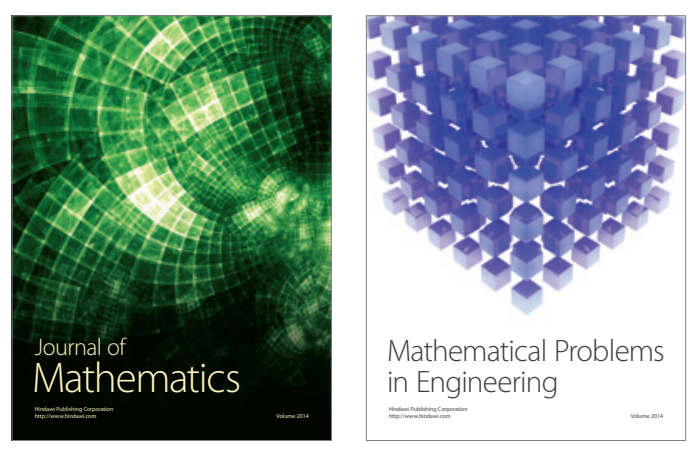

Mathematical Problems in Engineering
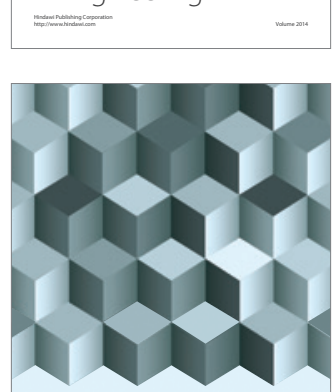

Journal of

Function Spaces
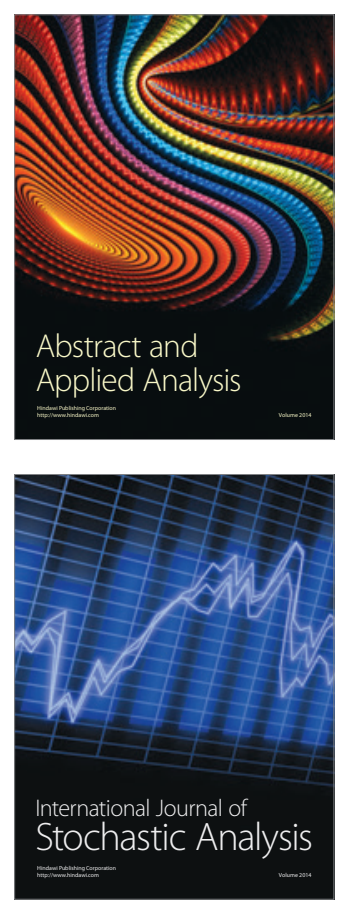

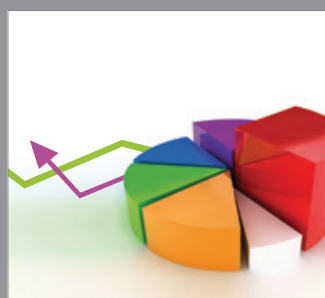

ournal of

Probability and Statistics

Promensencen
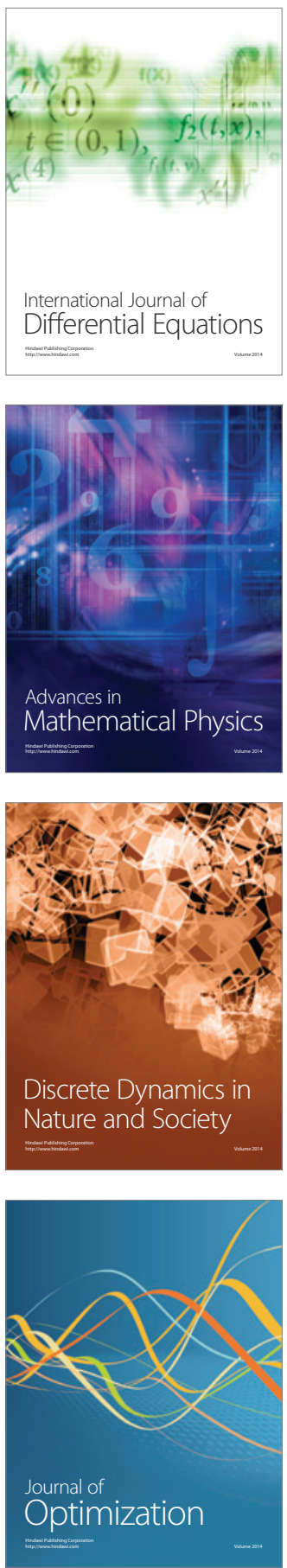